\title{
Prospects and Opportunities for Microsystems and Microfluidic Devices in the Field of Otorhinolaryngology
}

\author{
Se Hwan Hwang ${ }^{1,2}$ ([) · Alan M. Gonzalez-Suarez ${ }^{1}$ (D) · Gulnaz Stybayeva ${ }^{1}$ (i) $\cdot$ Alexander Revzin $^{1}$ (D) \\ ${ }^{1}$ Department of Physiology and Biomedical Engineering, Mayo Clinic, Rochester, MN, USA; ${ }^{2}$ Department of Otolaryngology-Head and Neck \\ Surgery, Bucheon St. Mary's Hospital, College of Medicine, The Catholic University of Korea, Bucheon, Korea
}

Microfluidic systems can be used to control picoliter to microliter volumes in ways not possible with other methods of fluid handling. In recent years, the field of microfluidics has grown rapidly, with microfluidic devices offering possibilities to impact biology and medicine. Microfluidic devices populated with human cells have the potential to mimic the physiological functions of tissues and organs in a three-dimensional microenvironment and enable the study of mechanisms of human diseases, drug discovery and the practice of personalized medicine. In the field of otorhinolaryngology, various types of microfluidic systems have already been introduced to study organ physiology, diagnose diseases, and evaluate therapeutic efficacy. Therefore, microfluidic technologies can be implemented at all levels of otorhinolaryngology. This review is intended to promote understanding of microfluidic properties and introduce the recent literature on application of microfluidic-related devices in the field of otorhinolaryngology.

Keywords. Microfluidics; Organoids; In Vitro Diagnostics; Organ-on-a-Chip; Otolaryngology

\section{INTRODUCTION}

During the past century, medicine experienced a remarkable growth in the knowledge of anatomy, physiology and pathology, leading to the development of new therapies. In the background of these improvements, there have been in vitro models using primary cell cultures and in vivo models using animal studies that have revealed the complex pathophysiology and human-related conditions [1,2]. However, there are gaps between physiology of human organs and in vitro cultures systems or animal

- Received April 13, 2020

Revised May 27, 2020

Accepted May 27, 2020

- Corresponding author: Alexander Revzin

Department of Physiology and Biomedical Engineering, Mayo Clinic, Rochester, MN 55905, USA

Tel: +1-507-293-2630

E-mail: revzin.alexander@mayo.edu

- Co-Corresponding author: Se Hwan Hwang

Department of Otolaryngology-Head and Neck Surgery, Bucheon St.

Mary's Hospital, College of Medicine, The Catholic University of Korea,

327 Sosa-ro, Wonmi-Gu, Bucheon 14647, Korea

Tel: +82-32-340-7044, Fax: +82-32-340-2674

E-mail: yellobird@catholic.ac.kr models (typically mice) used to mimic this physiology. For instance, primary cells quickly lose their tissue-specific functions when they are removed from living organisms and kept in standard culture conditions [1]. Thus, the investigation of single cells in highly artificial situations cannot reflect the characteristics of whole tissues [3]. On the other hand, animal models with induced human diseases are helpful models, but not completely accurate since pathologies are actually different among species. As a result, animal experiments suffer from significant biological differences, difficulty of translating animal-human data, prolonged processing time and costs, and ethical considerations [2].

During the past decade, several multidisciplinary research groups have engineered new methods to mimic the complexity of intra- and inter-organ interactions using artificial microenvironments called microfluidic systems. These systems allow the integration of human cells or tissues to imitate physiological conditions and functions of tissues and organs in a three-dimensional (3D) microenvironment, and replace or supplement animal experiments in disease-relevant tests or models [4,5]. Additionally, microfluidic systems present a variety of clinical and practical advantages for applications in medical field due to their extremely small sizes providing minimally invasive procedures,

Copyright $@ 2021$ by Korean Society of Otorhinolaryngology-Head and Neck Surgery

This is an open-access article distributed under the terms of the Creative Commons Attribution Non-Commercial License (https://creativecommons.org/licenses/by-nc/4.0)

which permits unrestricted non-commercial use, distribution, and reproduction in any medium, provided the original work is properly cited. 

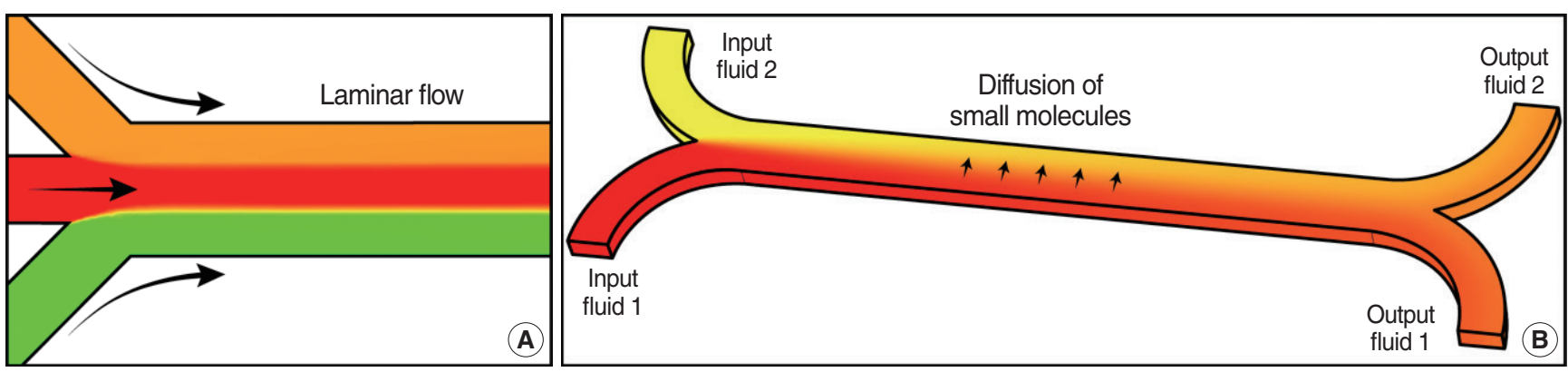

Fig. 1. Characteristics of flow in microfluidic devices. Laminar flow $(A)$ and diffusion $(B)$ of molecules inside microchannels are demonstrated.

low power and reagent consumption, fabrication processes with high reproducibility, and low cost per device, in conjunction with their multiple functionalities and compatibility with very large-scale integration electronics. High throughput screening and automation also becomes possible by the introduction of microfluidic systems [6]. In this review, we intend to help clinicians understand the basics of microfluidics by reviewing the recent literature about microfluidic devices in the field of otorhinolaryngology.

\section{OVERVIEW OF MICROFLUIDICS}

Microfluidics has been defined as the science and technology of systems that process or manipulate small volumes $\left(10^{-12}-10^{-6} \mathrm{li}-\right.$ ters) of fluids using channels with dimensions of tens to hundreds of micrometers [1,7]. At this length scale, the behavior of fluids is different compared to the intuitive macroscale. The flow is dominated by viscosity, instead of inertia, changing the governing physical effects of fluids in unique ways [8]. In contrast to macroscale where flow is often turbulent, at the microscale flow is often laminar with fluid traveling as consecutive layers that don't mix and move in a smooth and predictable direction (Fig. 1A). Due to laminar flow, diffusive mixing dominates over convective mixing (Fig. 1B). Another important effect is that surface

\section{H I G H L I G G H T S}

- Microfluidics can be used to guide small $\left(10^{-12}-10^{-6}\right.$ liters) volumes of liquid through channels with dimensions of tens to hundreds of micrometers.

- Microfluidic devices may be used to create three-dimensional cell cultures and may be automated to exercise spatiotemporal delivery of stimuli to these cultures.

- Microfluidic devices may contain different cell types for modeling heterotypic interactions within the same organ/tissue or between different organ models.

- Microfluidic devices may contain primary tissue for personalizing treatment options for patients. area relative to volume increases considerably, making capillarity force more significant than gravity inside microchannels, and making surface tension an important effect to consider [9].

These effects have been exploited to generate a wide variety of microfluidic devices for different applications. For instance, predictive laminar flow and diffusion can be used to generate concentration gradients across a culture chamber to generate a variable environment for chemotaxis studies [10-13]. Having a laminar flow can amplify the responsiveness of cells to a stimuli, by providing a constant inflow of stimulant closer to cells [14]. Surface tension effect can be finely controlled to generate monodisperse aqueous droplets immersed in oil in a high throughput manner which can be used for cell encapsulation, drug delivery or particle generation $[8,15]$. Capillarity phenomena can be used to passively pump fluid into microchannels [16,17]. Passive pumping is one of the simplest pumps and a basic component that can reduce the overall system size and maintain a stable and constant flow rate during long-term operation. Therefore, it has been widely studied in many applications, such as sensors, separators, mixers, and reactors [18].

Techniques for fabrication of microfluidic devices are already well established. Similar microfabrication techniques developed for the semiconductor industry are used to manufacture microfluidic devices. While a number of materials, including glass, thermoplastic polymers and hydrogels have been used for constructing microfluidic devices [19-24], silicone rubber or polydimethylsiloxane (PDMS) remains the material of choice for making microfluidic devices due to a relative ease and high fidelity fabrication, and because of its excellent biocompatibility $[25,26]$. PDMS has good oxygen diffusivity, $3.25 \times 10^{-5} \mathrm{~cm}^{2} / \mathrm{s}$ [27], which is slightly better than oxygen diffusivity in water, $1.96 \times 10^{-9} \mathrm{~cm}^{2} / \mathrm{s}$ [28]. This creates a suitable environment for long-term cell cultures inside PDMS-based microfluidic devices, even for cells with a high oxygen consumption rate (e.g., hepatocytes or pancreatic islets), without the need to deliver oxygen externally [14]. Furthermore, PDMS is optically transparent, enabling microscopic assessment of cell morphology or function over time. PDMS is a compliant material-an important feature that enables fabrication of microfluidic devices with automated, computer-controlled microvalves. These microvalves rely on fabrication of flexible thin 

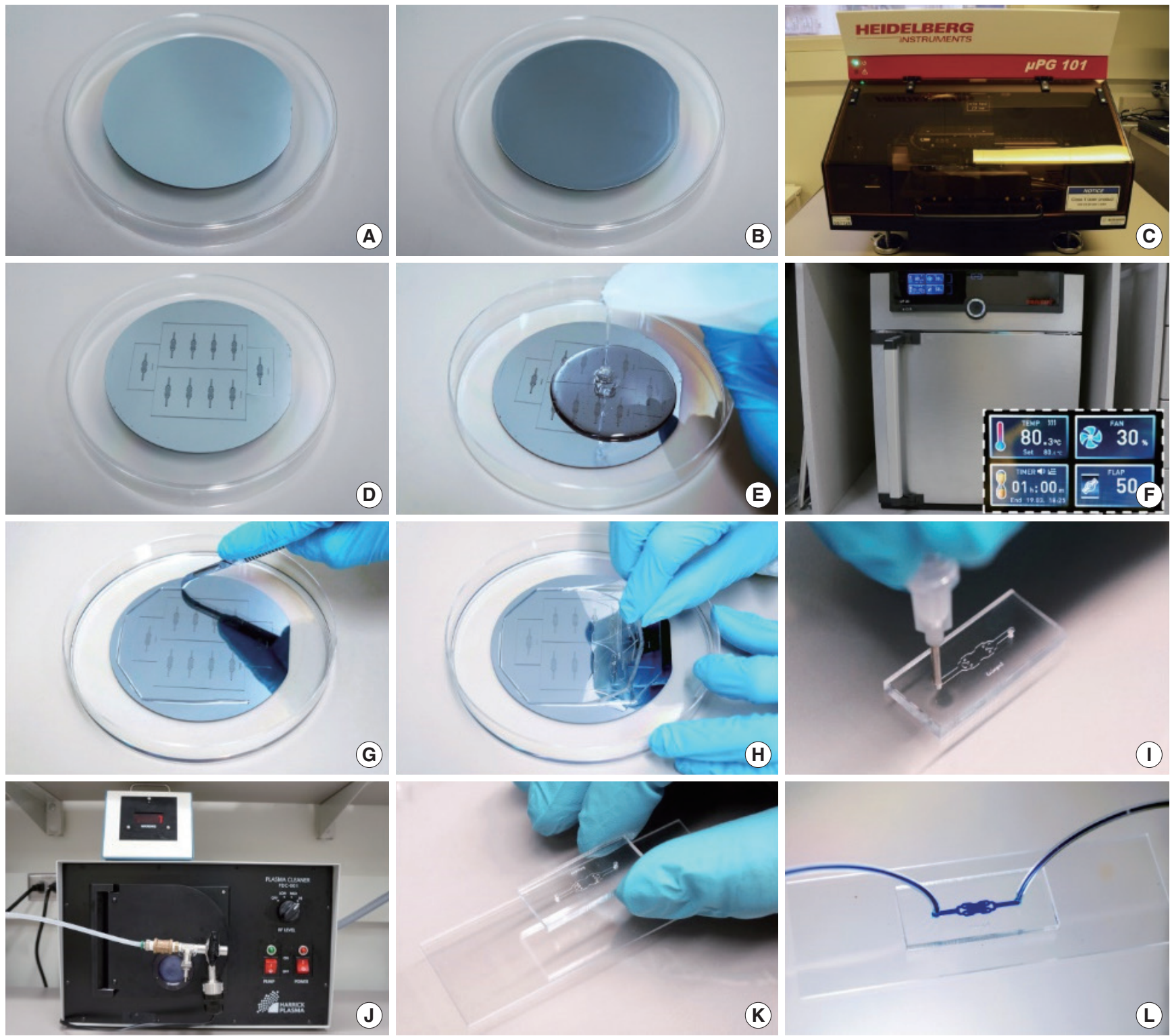

Fig. 2. Process of manufacturing a microfluidic device. A silicon wafer $(A)$ is coated with photoresist $(B)$ to transfer the design of a microfluidic device using standard photolithography techniques $(C)$. The mold $(D)$ is used as the frame to shape a polydimethylsiloxane (PDMS) mixture $(E)$ and baked to harden it $(F)$. Cured PDMS is cut $(G)$ and peeled off from mold $(H)$. After punching inlets and outlets (I), PDMS device and a glass substrate are treated with oxygen plasma $(\mathrm{J})$ to bond both surfaces $(\mathrm{K})$ and end up with the final microfluidic device $(\mathrm{L})$.

membranes that can be pneumatically actuated to block or permit flow in the microfluidic channels on demand [29]. Thus, PDMS-based microfluidic devices may be used to precisely control temporal delivery or metering of fluid over long periods [16].

To construct a PDMS microfluidic device, a master mold has to be fabricated first, to then cast PDMS replica with channels imprinted on the silicon rubber. The whole fabrication process, from mold to PDMS replica is described briefly and illustrated in Figs. 2 and 3. The first step is to design the microfluidic device in a computer using CAD (computer-aided design) software. Then, the device design is transferred to a silicon wafer (mold) using standard photolithography techniques. In this step, the silicon wafer is covered with a thin layer (can be from 1 to hundreds of micrometers, based on the specific application) of photoresist and then the designed is patterned on the photoresist using a ultraviolet light source. Unexposed photoresist is removed, resulting in the master mold. A liquid mixture of PDMS prepolymer and its curing agent is poured into the mold and heated in an oven to crosslink (harden) the PDMS. This process imprints the designed pattern on the bottom of the PDMS. After peeling the PDMS out of the mold, inlets and outlets are punched to connect the device to a pump or drainage system. Next, the PDMS 


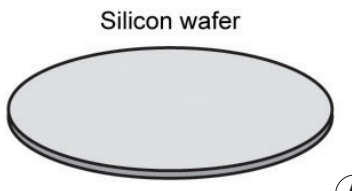

(A)

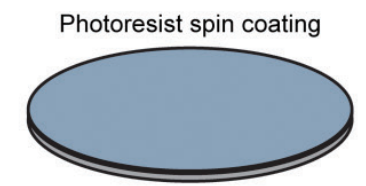

(B)

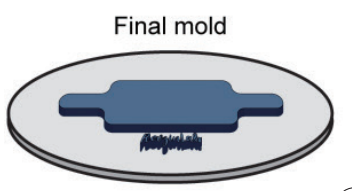

(E)

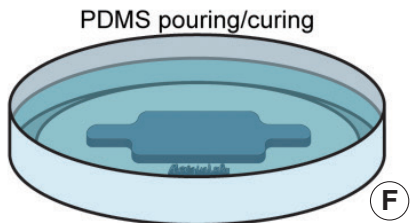

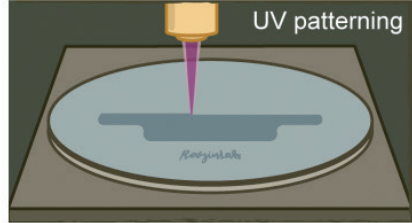

(C)

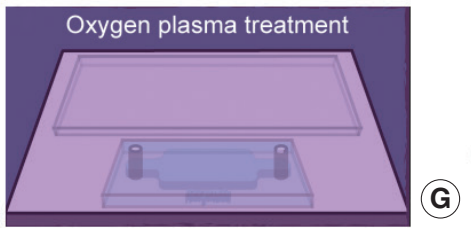

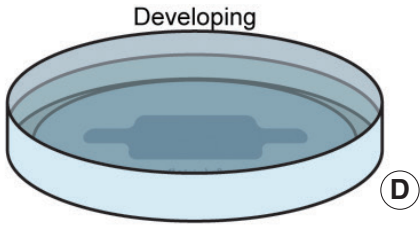

Final device

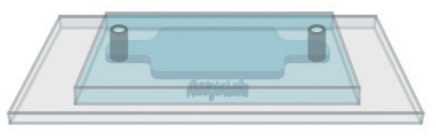

(H)

Fig. 3. Schematic depicting mold fabrication by photolithography and polydimethylsiloxane (PDMS) replicas fabrication by soft lithography. A clean silicon wafer $(A)$ is coated with photoresist $(B)$ and microfluidic device design is transferred by ultraviolet (UV) exposure (C). Unexposed photoresist is removed using a developer (D) to end up with the final mold (E). A PDMS mixture is poured on top of the mold (F), baked at $80^{\circ} \mathrm{C}$ for 1 hour, to then peel off the device from the mold. PDMS device and a glass substrate are plasma treated (G) and bond together to obtain the final device $(\mathrm{H})$.

device is bonded to a glass substrate that will serve as bottom of the microchannels by treating both surfaces with oxygen plasma. The plasma treatment oxidizes both surfaces and, upon contact, creates an airtight seal for the fluid microchannels [30]. Several PDMS layers can be assembled to create a more complex microfluidic device for different applications [31]. In recent years, the field of microfluidics has grown rapidly, and the advances made have generated great research interest in biology and medicine. Microfluidic devices for health care are highly anticipated in the following areas:

\section{Personalized medicine}

It is generally accepted that medications should better meet the needs of individual patients and their responses to treatment. There are reports of microfluidic devices that are populated with patient-derived cells. Such microfluidic devices have the potential to reproduce more accurately a physiological environment, allowing the formation of 3D organoids with patient-derived cells. Organoids can be exposed to different concentrations of a drug to evaluate efficacy in order to predict patient-specific responses and find a personalized therapy [32].

\section{Early diagnostics}

In almost all common diagnostic assays, incubation, labeling and washing are important steps than can be time consuming and expensive, due to large amounts of solutions needed to run assays in bulk. Due to the properties and size of microfluidic devices, these steps can be performed in a faster and cheaper way. Incubation times are reduced due to a fast transport of molecules in small channels. A multi-step assay can be automated incorporating microvalves on a microfluidic system and a personal computer. Since microchannels have very small volumes, the amount of solutions required for each assay is reduced. This will help in reducing the mortality rate and in controlling the propagation of life-threatening diseases such as malaria, HIV and
AIDS (human immunodeficiency virus infection and acquired immune deficiency syndrome), measles, tuberculosis, lower respiratory conditions and so on [33-35].

\section{High throughput mass screening}

Microfluidics is becoming increasingly appealing to mass screening due to recent trends in the biological sciences necessitating exceedingly large and comprehensive experiments [36]. Parallelization is possible in microfluidic devices, allowing to incorporate multiple assays in a single microfluidic device [37]. Variants of microfluidic and microfabrication approaches have been instrumental for sequencing human genome and analyzing biomarkers of diseases. Therefore, microfluidic devices can be used for point-of-care testing and on-time diagnosis of several diseases [38-43].

\section{Disease models or animal/patient-surrogates during drug development}

Major advances in human pluripotent stem cells (PSCs) have enabled the generation of 3D, in vitro, self-renewing, and self-organizing stem cell clusters in organoids. Organoid models derived from human PSCs can bridge the gap between traditional 2D culture models and animal studies by reproducing the specific traits of tissues or organs. Stem cells and patient-induced PSCs could be a great tool for studying the mechanisms of human disease and drug development, being a currently growing research field (Fig. 4) [44].

\section{CURRENT STUDIES ABOUT MICROFLUIDIC DEVICES IN OTORHINOLARYNGOLOGY}

Microfluidics technologies occupy an important niche between in vitro culture systems that are too simplistic for modeling human diseases and animal models that are too complex to untan- 


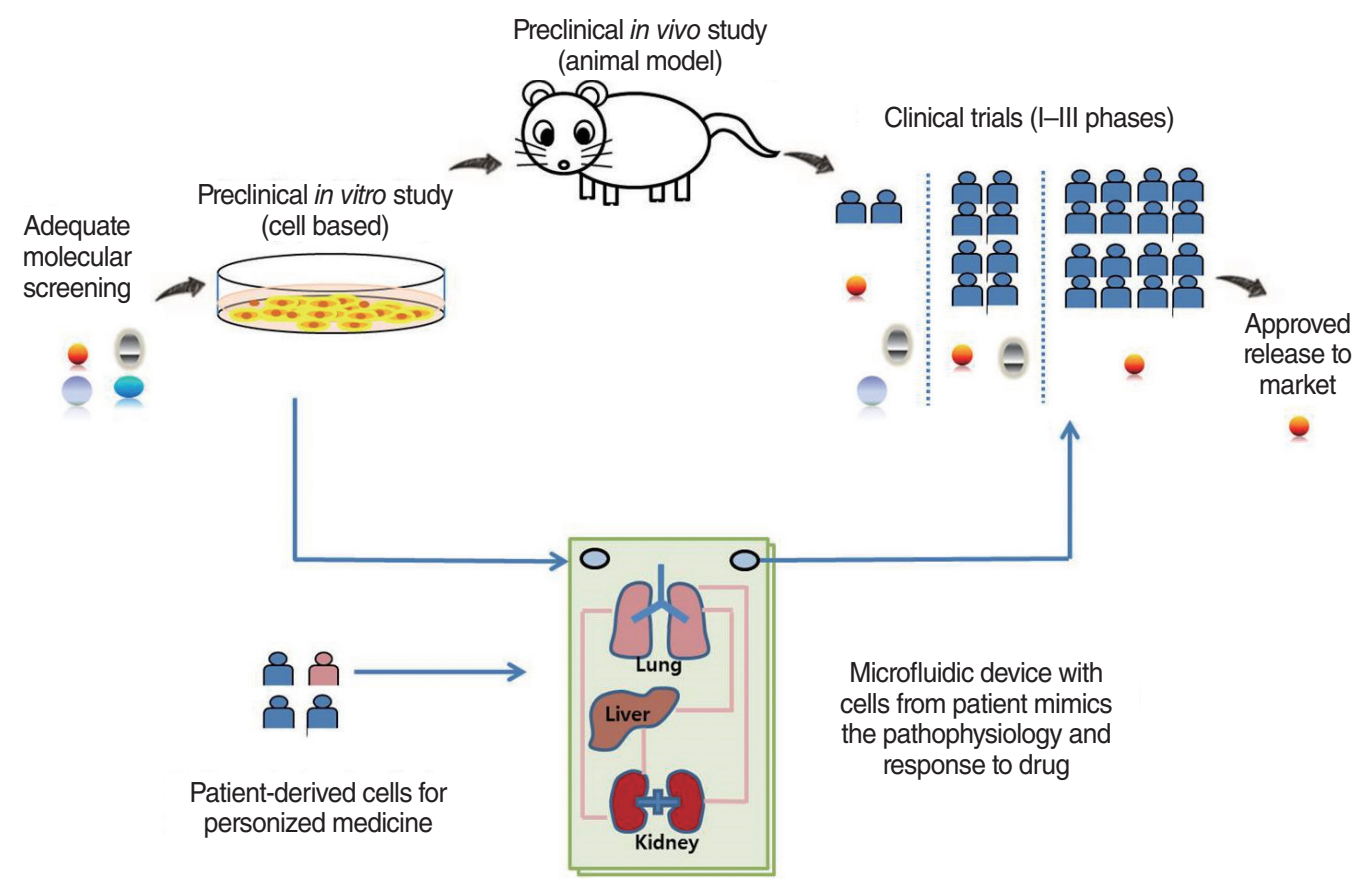

Fig. 4. Schematic illustration of organoid models. Microfluidic devices containing patient-derived cells could be used to evaluate drug efficacy, eliminating the need for animal models and enabling the practice of personalized medicine.

gle mechanisms that underlie disease progression. In addition, microfluidic devices offer excellent prospects for miniaturizing, expediting and automating assays for disease diagnosis. In particular, considering that otorhinolaryngology field is small but complex and diverse, filled with specialized tissues and functions, microsystems and microfluidic devices offer tremendous potential to improve the field.

For example, microfluidic aqueous-aqueous interface with an ultra-low interfacial tension was reported to respond to sound of different frequencies and amplitudes [45], which could represent a new type of cochlear implant to sense and transmit vibrations induced by sound. On the other hand, microfluidic devices populated with cells from patients with allergic rhinitis, like an in vitro model of human nasal mucosa, could be used to mimic allergic reactions or drug responses in real-time. Microfluidic devices with nasal epithelial cells or immunocytes could be perfused with allergens or drugs to reflect the response of such cells in terms of quantity of cytokine secretion.

\section{Rhinology}

Asthma and allergic rhinitis are caused by exaggerated immune responses, including the formation of specific immunoglobulin $\mathrm{E}$ (IgE)-antibodies upon allergen exposure, the formation of IgEantibodies that bind to mast cells and basophils, and the activation of immune cells by allergens [46]. The skin prick test is an important diagnostic tool for allergen sensitization, but it is implicated in systemic reactions and drug interactions. In contrast, in vitro serum testing for specific $\operatorname{IgE}$ antibodies is convenient, completely removing the risk of an anaphylactic reaction [47]. This test is usually performed using the well-known enzymelinked immunosorbent assay (ELISA). A previous report shows a comparison between a microfluidic cartridge-based system using a colorimetric ELISA test, to a skin prick test to diagnose inhaled allergen sensitization in patients. The microfluidic device was covered with a nitrocellulose membrane containing an antigenic protein and was placed in the center of the cartridge, where, upon deposition of sample in the cartridge, IgE present in serum bonded to the antigenic proteins. Adding a substrate resulted in a colorimetric reaction, where color intensity was proportional to amount of antigen-antibody complexes in the membrane. Serum-specific IgE tests using the microfluidic cartridge-based system showed moderate to high accuracy in diagnosing house dustmite sensitization (sensitivity, 48\%-77\%; specificity, 64\%-95\%) $[48,49]$.

Another important subject is allergies detection. A common method for this is allergy blood testing, where allergen-specific antibodies are detected on serum using multiple immunoassays, one for each allergen. A research group used commercially available microarrays to demonstrate an integrated microfluidic system that automates the entire process of allergy detection, including hybrid detection, mixing, reagent delivery, and washing. Using their microfluidic platform, operating time was reduced by about $30 \%$ compared to manual processing, and the sample and reagent consumption was reduced by $25 \%$ [50]. They suggested that microfluidic array systems overcome the limitations of existing technologies by offering ease of use, fast analysis, 
minimal sample requirements, reduced waste generation, and minimal exposure to hazardous substances [38,51].

Basophils play an important role in IgE-mediated allergic reactions caused by re-exposure to certain allergens. Similar to mast cells, basophils have high-affinity receptors (FceRI) to IgEantibodies on their surface [52]. After activation, these cells degranulate and release different immune modulators, such as histamine, by cross-linking allergens to IgE-antibodies (Fig. 5) [53].
Because basophils can be more easily separated from blood than mast cells in tissues, a functional cell-based assay, the basophil activation test (BAT), is used to assess IgE-mediated allergic responses by measuring released allergen responses, such as histamine, or the expression of the markers CD63 and CD203c at the cell surface (Fig. 6) [54]. In addition, BAT can be used to monitor the clinical response to allergen-specific immunotherapy and other immunomodulatory treatments $[55,56]$. However,
Resting basophil

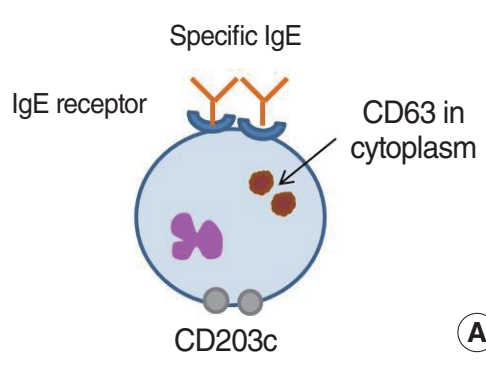

Activated basophil

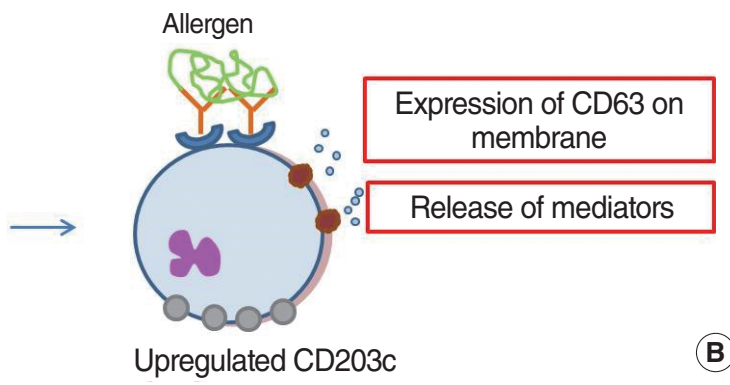

Fig. 5. Schematic illustration of basophil activation. Resting basophils (A) release various immune modulators, such as histamine, and express CD63 and CD203c at the cell surface after activation (B) by cross-linking allergens and immunoglobulin E (lgE)-antibodies.

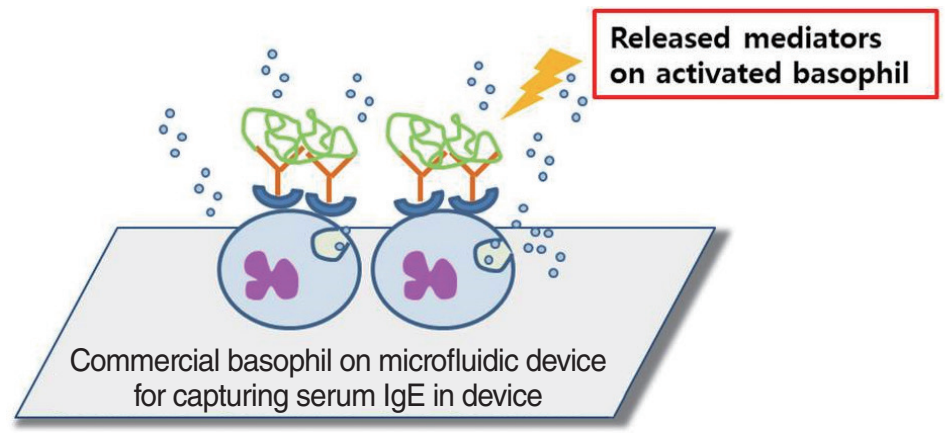

Utilization of specific IgE from patient's serum

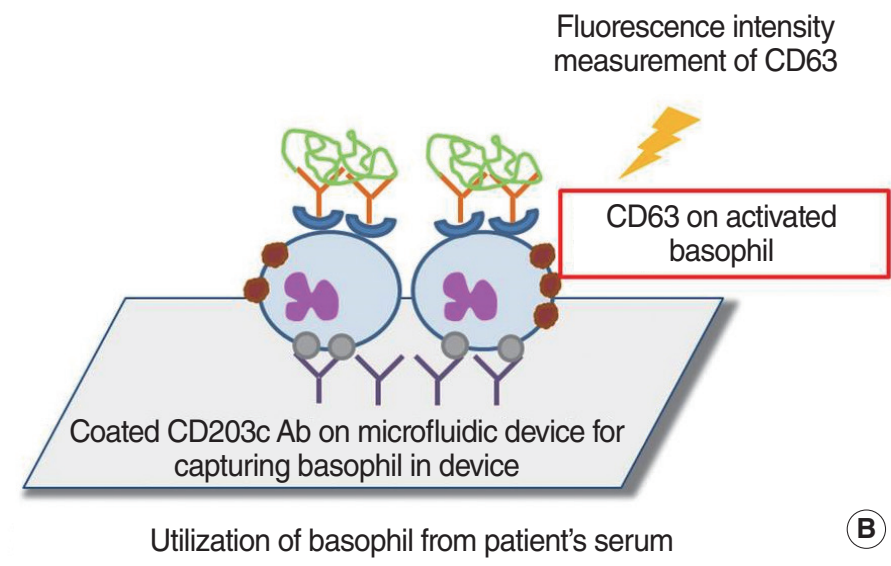

Fig. 6. Principles of basophil activation test in microfluidic devices. (A) The dye-loaded commercial basophils are sensitized using a patient's serum-specific immunoglobulin $(\mathrm{lg})$. After challenge with an allergen, the dye fluorescence is secreted from the basophils and analyzed in the detection chamber. (B) The microfluidic device captures basophils with anti-CD203c antibody and measures the level of CD63 expression in the captured allergen-exposed basophils. 
this test is flow-cytometry-based, making it time consuming and costly, and technical requirements for performance are difficult to meet clinically [56].

To measure allergic degranulation in living cells, previous studies have demonstrated an integrated platform called lab-on-a-disk (LOAD), a centrifugal microfluidic setup that looks like a CD, that can measure degranulation or exocytosis from basophils [35]. In this device, a specific dye (acridine orange) can be loaded into basophilic granules to determine the degree of degranulation compared with a control value. Since the LOAD device works by spinning, centrifugal force drives reagents into different chambers at specific time points for reaction development, without the need of a micro pump for liquid handling. For this test, basophils were exposed to acridine orange to label granulocytes and allow the observation of the degranulation process. After basophils sensitized with patient serum (including specific IgE-antibodies) were exposed with the suspected allergens, basophils secreted the granules contents into the extracellular space. The fluorescence intensity of the media was analyzed to assess the effect of allergen on basophils. That LOAD platform demonstrated some advantages over the standard test, such as shorter analysis time, improved sensitivity, simple procedure, lower cost, and reduced consumption of samples and reagents (Fig. 6A) [57,58].

Aljadi et al. [59] designed a device that captures basophils directly from whole blood, as an alternative way to assess effect of allergens on a blood sample. CD63 is located in the membranes of intracellular secretory granules in resting basophils. After stimulation by Fc\&RI, secretory granules fuse with the plasma membrane and CD63 is expressed on the surface of the degranulating basophils [60]. To capture basophils, Aljadi et al. [59] coated the surface of a microfluidic device with anti-CD203c antibody to capture activated basophils after exposure of samples to allergens (Fig. 6B). Then, CD63 expression was assessed in captured basophils by fluorescence and directly compared to a healthy control. A conventional flow cytometry analysis of basophil activation was also performed for comparison. The CD63 expression in the anti-FceRI-activated basophils captured by the microfluidic chip was significantly higher in allergic patients than in the healthy control group $(P=0.03)$. In addition, the results obtained using the microfluidic platform did not differ significantly from flow cytometry results.

It is known that nasal mucosa is the first line of defense against air pollutants and microorganisms that can start an allergic or immune reaction, among other diseases. There is a high interest in studying nasal mucosa because it could reveal the pathophysiology of many respiratory diseases [61]. In 2017, Na et al. [62] introduced a new in vitro model of human nasal mucosa by imitating the complex cell-extracellular matrix (ECM) interaction and structure under air-liquid interface culture conditions. The authors designed an microfluidic device with three chambers: a ECM chamber in the middle, a nasal epithelial monolayer on one side and an endothelial monolayer on the other side (Fig. 7). During cell culture, nasal layer formed glandlike structures, successfully mimicking in vivo nasal mucosa. The
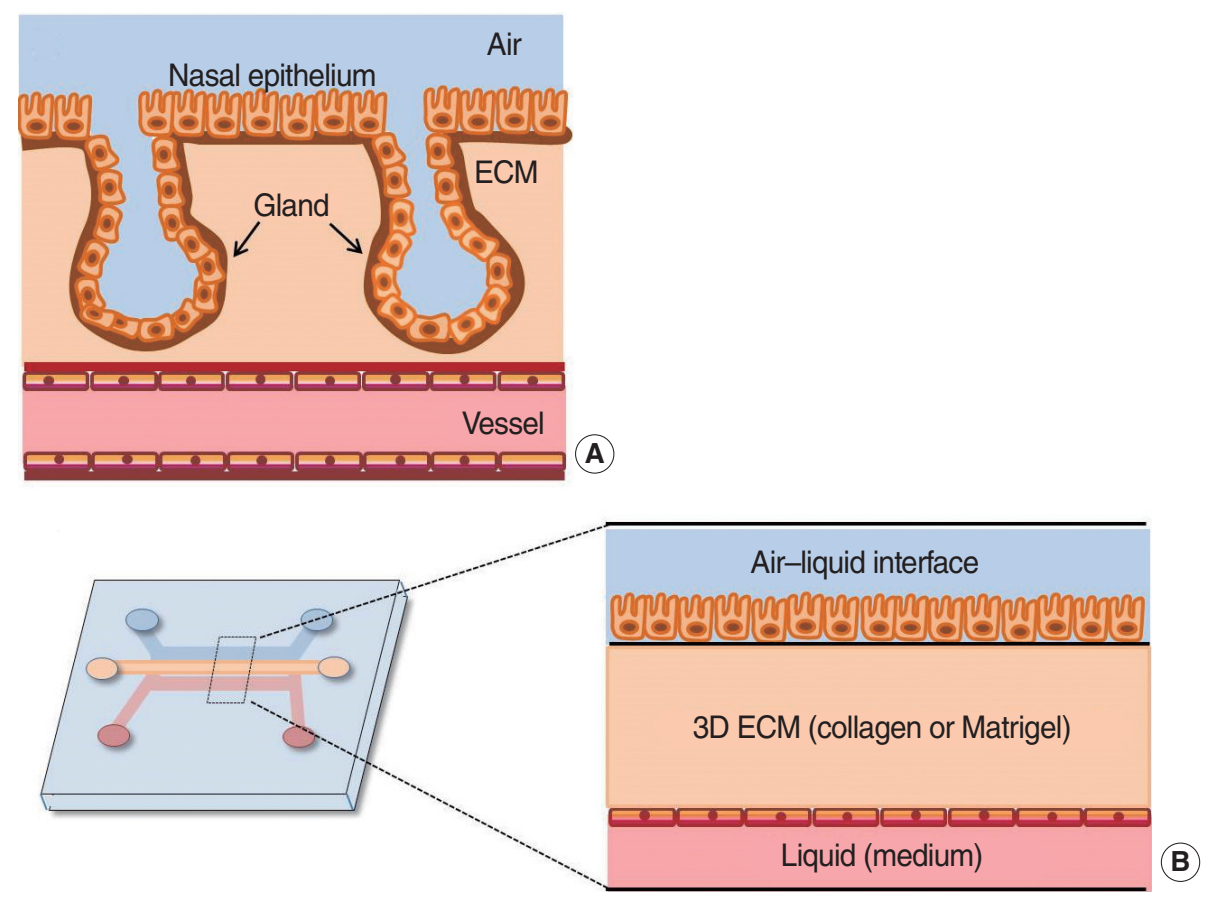

Fig. 7. Microfluidic device to mimic the structure of nasal mucosa. (A) Nasal mucosa structure has three layers (epithelial layer, extracellular matrix, and vascular layer). (B) Each layer is imitated using an air-liquid channel, gel (collagen or Matrigel)-filled channel, and liquid-covered channel, respectively. ECM, cell-extracellular matrix; 3D, three-dimensional. 
function of the mucous glands was identified by immunofluorescent staining of the mucin protein (MUC5b) and the gland development marker (Sox9).

Cilia movement and the production of mucus by the nasal mucosa are a unique defense mechanism to protect the airway from external stimulants [63]. In a previous study, in vitro differentiated human nasal epithelial cells were incorporated into a microfluidic device for real-time monitoring of cilia beating frequency, as way to test the response of the human airway to drugs or toxic agents [64]. Cilia beating frequency was monitored by placing a cilia-embedded microchip on the microscope (Fig. 8) The responsiveness of the ciliated cells to dynamic exposure of formaldehyde gas was monitored and analyzed simultaneously. The researchers reported that formaldehyde had an irritating effect on epithelial cells and that the frequency of ciliary beats could be a sensitive indicator of toxic inhalation in human cells.

The developed systems and experimental models are more realistic for clinical applications than conventional cell culture techniques considering the possibility to incorporate different cell types and components, and the ability to perform immunos-

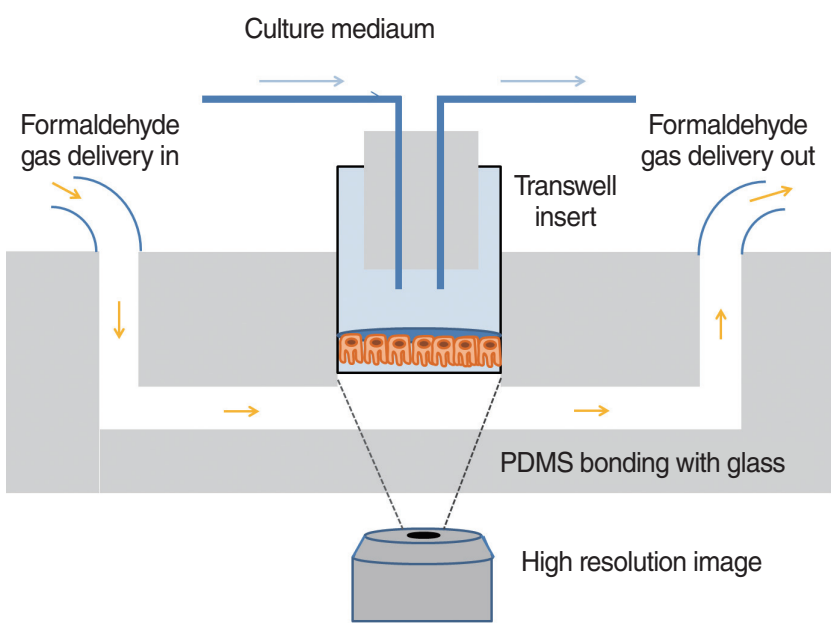

Fig. 8. Real-time monitoring of cilia beating frequency in microfluidic device. In vitro differentiated human nasal epithelial cells are incorporated into microfluidic chips for real-time monitoring of the cilia beating function. PDMS, polydimethylsiloxane. taining in situ. These types of models developed in microfluidic devices could be used as more accurate physiological model to study diseases related to rhinology.

\section{Otology}

One of the main targets of drug delivery for this disorder is sensory neurons in the cochlea [65]. However, treatment of hearing impairment is challenging because local entry of systemic circulating drugs is hampered by the blood-cochlear barrier, with cochlear blood supply estimated to represent less than one-millionth of total cardiac output in humans [66]. Additionally, many of the relevant drugs and drug candidates cause significant side effects when delivered systemically. For this reason, there is an interest on delivering drugs directly into the cochlea; in this way, side effects could be minimized, there could be an improved access to cells of interest and a significantly increase of target specificity [67].

Currently, access to the inner ear is typically obtained using a middle ear delivery system in which a drug is deposited on the round window membrane (RWM) in a reservoir, such as a gel or microparticle formulation, and then transported through the membrane into the cochlea. However, wide patient-to-patient variability in the mechanical and transport properties of the RWM causes a high degree of uncertainty and variability in drug concentration and delivery profiles [68]. More direct methods, such as the use of osmotic pumps, provide a limited delivery period and do not allow real-time control of the delivery profile [69]. Therefore, a new approach based on canalostomy and active micropumps is being considered to reduce downstream hydraulic resistance and enable deep penetration of medication into the apical zone. Nevertheless, because the volume of the scalar tympani is about $30 \mu \mathrm{L}$ and the hair cells inside the Corti organ are very delicate, rapid infusion of fluid into this space can increase pressure and cause permanent damage [65]. To prevent those complications, additional surgical entry points are necessary, which can increase the risk and difficulty of surgery (Table 1 ).

Recently, reciprocating micropumps based on microfluidics/ microelectromechanical systems (MEMS) technologies have been developed for safe and effective drug delivery, allowing di-

Table 1. The merits and demerits of direct inner-ear drug delivery methods

\begin{tabular}{|c|c|c|}
\hline Delivery method & Merit & Demerit \\
\hline Intratympanic injection & Clinically accepted, minimally invasive & $\begin{array}{l}\text { Rapid clearance through Eustachian tube, relies } \\
\text { on diffusion through RWM }\end{array}$ \\
\hline $\begin{array}{l}\text { Passive release degradable reservoir (drug) at } \\
\text { RWM }\end{array}$ & $\begin{array}{l}\text { Prolonged residual time at RWM, minimally } \\
\text { invasive, currently in clinical trials }\end{array}$ & $\begin{array}{l}\text { Dependent on diffusion through RWM } \\
\text { Variability from patients to patients }\end{array}$ \\
\hline Intracochlear osmotic pump & Extended drug delivery to inner ear fluid & Lack of control over release profile, highly invasive \\
\hline Intracochlear active pumping with canalostomy & Superior pharmacokinetic distribution in cochlea & Highly invasive with multiple surgical site \\
\hline Cochlear prosthesis (implant)-mediated delivery & $\begin{array}{l}\text { Integrated with well-established therapy, } \\
\text { enhances clinical benefit of implant }\end{array}$ & Limited to cochlear implant candidates only \\
\hline
\end{tabular}

RWM, round window membrane. 
rect injection into the inner ear with low mechanical and fluid fluctuations in the cochlea [65]. This is achieved by periodically infusing and withdrawing a constant volume of fluid in a process designed to improve drug mixing and peak transport. The infusion part of the cycle lasts a few seconds and can pump an amount lower than $1 \mu \mathrm{L}$. The rest of the cycle lasts for several minutes and returns the mixture of endogenous perilymph and drug back into the device (Fig. 9). To prevent damage caused by flow, the infusion withdrawal cycle can be carried out at very low flow rates and deliver drug solutions without causing net volume changes. In addition, using a single cannula for both infusion and withdrawal can reduce biofouling by maintaining positive pressure at the outlet during the whole cycle [67].

Animal models have been used to analyze the efficacy and safety of reciprocating micropumps. The ionotropic receptor antagonist DNQX (6,7-dinitroquinoxaline-2,3-dione) is used as a physiological indicator of drug location, and auditory brain stem response $(\mathrm{ABR})$ measurements at decreasing frequencies can be used to estimate how far the drug has moved along the tonotopic axis. Distortion product otoacoustic emissions (DPOAE), on the other hand, is not sensitive to DNQX and is useful as a control to provide an estimate of the damage caused by surgery [70]. In previous studies, DPOAE was not significantly affected by surgery or device activation, and ABR threshold elevation was reversibly induced by DNQX. Those results indicate that the reciprocating drug delivery devices offer significant safety and effectiveness over a short period of time [70-72].

Dizziness and imbalance are common in older people and can significantly affect quality of life. Dizziness interferes with the daily activities of many people older than 70 years [73]. People suffering from vestibular-related disorders could benefit from the development of vestibular nerve prostheses, which bypass the dysfunctional elements in the vestibular pathway using artificial stimuli. Recent work has tested an alternative approach to
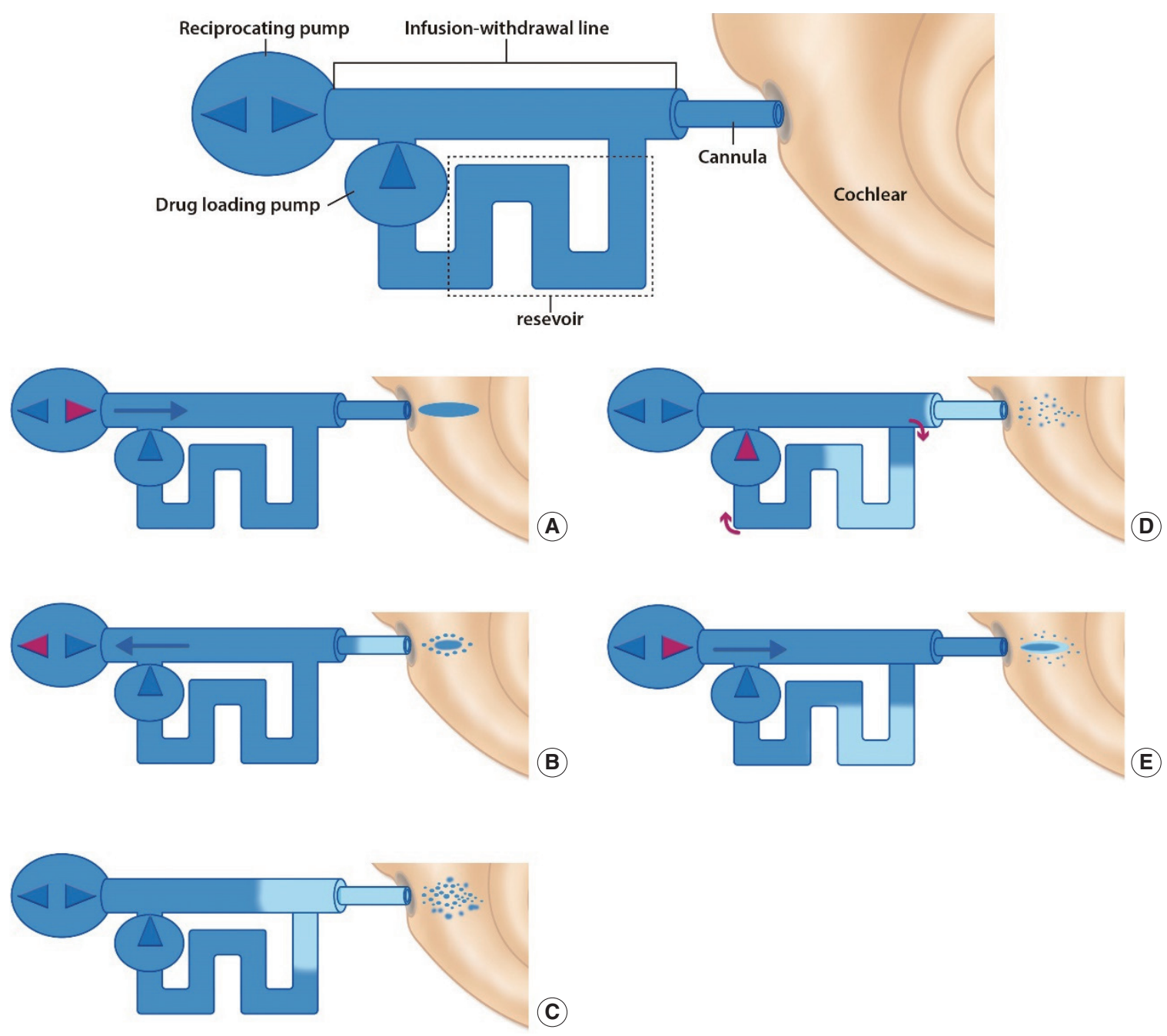

Fig. 9. Schematic illustration of reciprocating system and sequential operation. The red pump indicates an actively working pump in each individual step (alphabetical order, A-E). The blue points near the cannula indicate the drug spread by diffusion. Light color changes represent the drug diluted by perilymph. 
angular velocity sensing based on the way the natural vestibular semicircular canal works, whereby the inertial mass of a fluid is used to modify the sensing structure during rotation [74]. The device was manufactured using a commercially available MEMS process. The microfluidic channel consisted of an etched glass layer sandwiching a bulk-microfabricated silicon substrate containing an etched structure. The size of the device was $6 \mathrm{~mm} \times$ $6 \mathrm{~mm}$, including the pad area, and it used only $300 \mu \mathrm{W}$ at $2 \mathrm{~V}$ and showed an angular velocity sensitivity of less than $1 \% \mathrm{sec}$, which is similar to that of the natural vestibular system [74]. This result suggested the potential for the near-term development of a fully functional vestibular implant.

\section{Head and neck}

Head and neck cancers are the sixth most common cancers in the world, with an incidence of about 600,000 cases each year. Despite advanced surgical and medical treatment strategies, the overall survival rate of patients has remained unchanged for decades $[75,76]$. One reason for that static survival rate from head and neck cancers is considered to be the significant genetic heterogeneity of the disease. In particular, metastatic lesions can contain different genetic mutations that cause growth, potential therapeutic resistance, and relapse [77]. Traditional tissue biopsies can provide histological definition and show the genetic profile of a cancer, but they can be too difficult or invasive to perform in recurrent or anatomically dangerous lesions or too risky for patients to experience sequentially. In addition, "spatial heterogeneity" can produce sampling bias, making samples inadequate because they do not represent the entire mutation [78].

Recent studies focused on providing intact single cells that can be further characterized to provide protein expression- and gene-level data. Circulating tumor cells (CTCs) are cells derived from tumor masses (primary or metastatic) that have entered the vascular circulation. They represent metastatic seeds and provide a window into metastasis. CTCs are thus likely to provide important information about the metastatic cascade and tumor heterogeneity and chemistry [77]. Exosomes are small (diameter 50-100 nm) vesicles secreted from various mammalian cells. Because exosomes are released through fusion between the endosome membrane compartment and the plasma membrane, extracellular and intracellular biomarkers can reveal the type and state of the cell of origin (Fig. 10). Tumor cell-derived exosomes can activate tumor progression and metastasis, and they contain specific messages that have been investigated by diagnostic and therapeutic researchers [79].

Liquid biopsies provide an opportunity to detect, analyze, and monitor cancer in various body drainages, such as blood or saliva, instead of cancer tissue fragments. They consider biological matrices such as CTCs, cell-free nucleic acids, exosomes, or "tumor-educated platelets." In addition to offering a noninvasive or minimally invasive procedure, liquid biopsies are also expected to offer a better view of tumor heterogeneity than tradi-

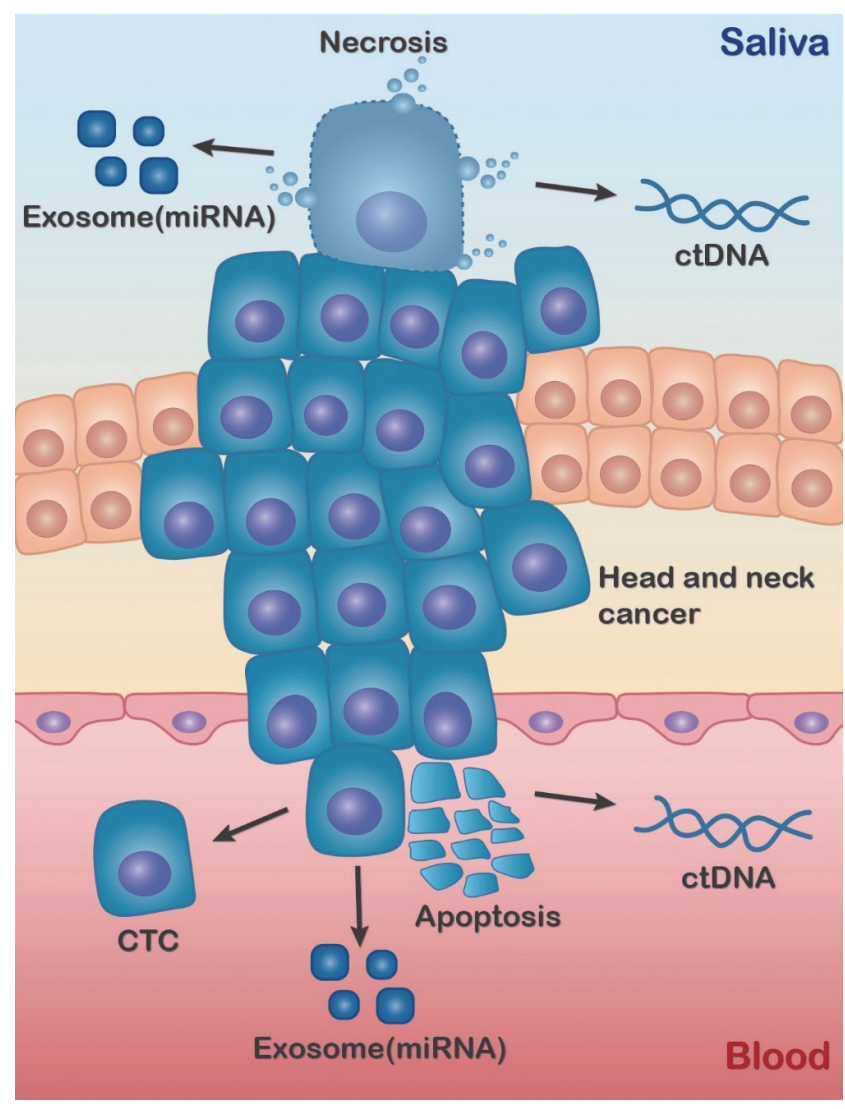

Fig. 10. Circulating biomarkers released from cancer tissue. Apoptotic cancer cells release biomarkers (circulating tumor cells [CTCs], circulating tumor DNA [ctDNA], and exosomal microRNA [miRNA]) into blood, whereas necrotic tumor cells shed biomarkers into saliva.

tional biopsies and be able to monitor the evolution of cancer in real time. However, the half-life of CTCs is estimated to be less than a few hours due to the shear force of turbulent blood flow and the immunological monitoring of circulation [77]. Because the yield of CTCs is tens to hundreds per milliliter of blood, they account for $<0.004 \%$ of all monocyte cells, or approximately 1 in 1 billion circulating cells. Therefore, enriching and sequestering CTCs are important challenges [77]. Also, conventional methods for isolating and analyzing high-purity exosomes in clinical settings have many disadvantages, including low yield and purity, long processing time, high cost, and difficulty of standardization [80].

Microfluidic devices have enabled advances in the purification and analysis of liquid biopsy components [81]. These devices have been designed to capture CTCs by surface markers or isolate them based on physical properties (for example size). Enriched cells undergo an antibody staining protocol to accurately identify CTCs. The accepted definition of CTCs in head and neck cancers is cell staining that is positive for epithelial markers and DAPI (4',6-diamidino-2-phenylindole; nucleus) and negative for CD45 (a leukocyte marker). Cytokeratin 8, 18, 19 or 20 also appear to be reliable in head and neck cancers. In addition, 
markers for mesenchymal (N-cadherin or vimentin) or stem cell (CD133) phenotypes have been applied [82,83]. Numerous marker-based assays using microfluidic techniques are available and have been reported previously $[84,85]$. Based on the microfluidic technique, automated exosome separation into a single device avoids multi-step protocols that require multiple instruments and skilled technicians. Shorter isolation times (10-20 minutes of treatment time) and low sample consumption enhance biosafety and enable high throughput screening in many patient populations [77].

Patient-derived xenograft models offer the advantage of closely reproducing human in vivo models for various therapeutic and test purposes. However, it can take up to 6 months to produce xenografts, the process costs thousands of dollars and uses a lot of animals, and the tumor is inevitably affected by the rodent host [86]. Microfluidic culture of patient-derived tumor tissue has the potential to more closely regenerate the tumor microenvironment [87]. By continually delivering nutrients and removing waste from tissues while maintaining complex multicellular structures without rodent factors, microfluidic technology offers unique properties that could make it a platform for preclinical biological investigations [88]. In previous microfluidic irradiation models with metastatic lymph nodes and primary tumors from different patients, immunohistochemistry expression profiles relevant to cell death and proliferation markers were measured to determine an individual's tumor response to irradiation. The results showed clear inter- and intra-patient variability in response to irradiation when measuring a variety of parameters, which offered the potential to predict patient responses and could be helpful in predicting treatment effectiveness and quality of life, as well as offering cost savings and improved patient care [89].

Additionally, based on the concept that the efficacy of immunotherapy is related to immune cell migration to cancer cells, a humanized in vitro microfluidic chip assay was manufactured to test immunotherapeutic drugs against patient samples (Fig. 11) [90]. In that device, the patient's own serum was used instead of the commonly used fetal bovine serum to mimic the in vivo interaction between cancer and immune cells. Immune cell migration toward cancer cells was used as a parameter for the efficacy of immunotherapy against cancer cells [76]. Interestingly, the results indicated between-patient variability in two agents that induce immune cell migration toward cancer cells. Thus, this assay could be used to predict the efficacy of immunotherapeutics for individual patients [90].

\section{CONCLUSION}

In this review, we provided a brief explanation of techniques for fabricating microfluidic devices and offered examples of such devices being applied in the field of otorhinolaryngology. Over-

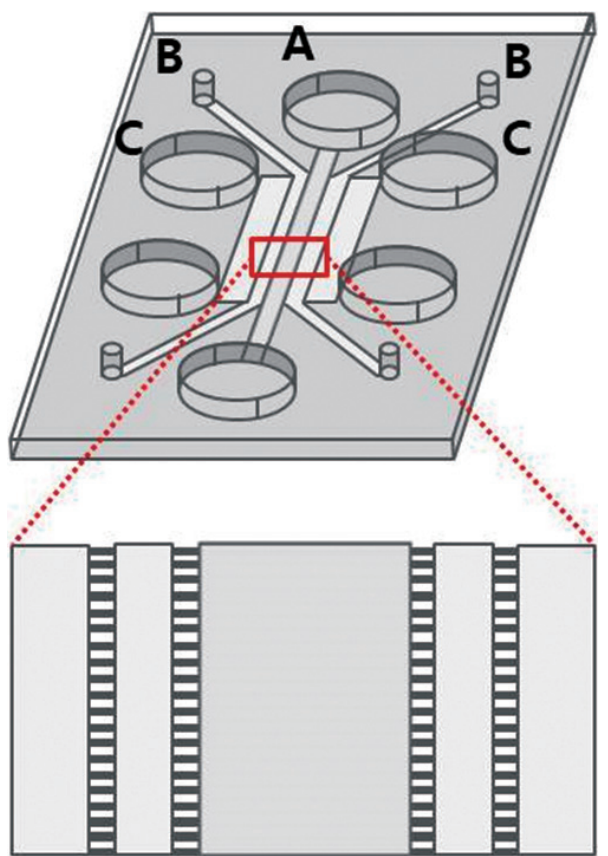

Fig. 11. Microfluidic chip design. Immune cells are loaded into Channel $A$, and cancer cells are place in Channel $B$. Channel $C$ is used for hydration. The red rectangle magnifies the connections between channels, which are linked with microtubules.

all, microfluidic systems represent a novel means of recapitulating complex human tissue microenvironments and are increasingly being used for personalizing patient treatment. Therefore, this technology deserves to be on the radar of clinicians/scientists in otorhinolaryngology.

\section{CONFLICT OF INTEREST}

No potential conflict of interest relevant to this article was reported.

\section{ACKNOWLEDGMENTS}

The authors would like to thank the members of the Cellular Microsystems and Biosensors Laboratory at Mayo Clinic, Rochester, MN, USA. This research was supported by the Basic Science Research Program through the National Research Foundation of Korea (NRF) funded by the Ministry of Education (2018R1D1A1B07045421). Additional support was provided by the National Institutes of Health (DK107255).

\section{ORCID}

Se Hwan Hwang https://orcid.org/0000-0002-2838-7820 
Alan M. Gonzalez-Suarez

https://orcid.org/0000-0001-6442-4353

Gulnaz Stybayeva

Alexander Revzin

https://orcid.org/0000-0002-1453-245X

https://orcid.org/0000-0002-8737-3250

\section{AUTHOR CONTRIBUTIONS}

Conceptualization: SHH, AR. Data curation: SHH, AMGS, AR. Formal analysis: SHH, GS, AR. Funding acquisition: SHH, AR. Methodology: SHH, AMGS, AR. Project administration: SHH, GS, AR. Visualization: SHH, AMGS, AR. Writing-original draft, review, \& editing: all authors.

\section{REFERENCES}

1. Lee PJ, Hung PJ, Lee LP. An artificial liver sinusoid with a microfluidic endothelial-like barrier for primary hepatocyte culture. Biotechnol Bioeng. 2007 Aug;97(5):1340-6.

2. Geraili A, Jafari P, Hassani MS, Araghi BH, Mohammadi MH, Ghafari AM, et al. Controlling differentiation of stem cells for developing personalized organ-on-chip platforms. Adv Healthc Mater. 2018 Jan; 7(2):1700426.

3. Kieninger J, Weltin A, Flamm H, Urban GA. Microsensor systems for cell metabolism: from 2D culture to organ-on-chip. Lab Chip. 2018 May;18(9):1274-91.

4. Huh D, Torisawa YS, Hamilton GA, Kim HJ, Ingber DE. Microengineered physiological biomimicry: organs-on-chips. Lab Chip. 2012 Jun;12(12):2156-64.

5. Park H, Lim DJ, Sung M, Lee SH, Na D, Park H. Microengineered platforms for co-cultured mesenchymal stem cells towards vascularized bone tissue engineering. Tissue Eng Regen Med. 2016 Oct;13(5): 465-74.

6. Gurman P, Miranda OR, Clayton K, Rosen Y, Elman NM. Clinical applications of biomedical microdevices for controlled drug delivery. Mayo Clin Proc. 2015 Jan;90(1):93-108.

7. Whitesides GM. The origins and the future of microfluidics. Nature. 2006 Jul;442(7101):368-73.

8. Beebe DJ, Mensing GA, Walker GM. Physics and applications of microfluidics in biology. Annu Rev Biomed Eng. 2002;4:261-86.

9. Atencia J, Beebe DJ. Controlled microfluidic interfaces. Nature. 2005 Sep;437(7059):648-55.

10. Li Jeon N, Baskaran H, Dertinger SK, Whitesides GM, Van de Water L, Toner M. Neutrophil chemotaxis in linear and complex gradients of interleukin- 8 formed in a microfabricated device. Nat Biotechnol. 2002 Aug;20(8):826-30.

11. Walker GM, Sai J, Richmond A, Stremler M, Chung CY, Wikswo JP. Effects of flow and diffusion on chemotaxis studies in a microfabricated gradient generator. Lab Chip. 2005 Jun;5(6):611-8.

12. Agrawal N, Toner M, Irimia D. Neutrophil migration assay from a drop of blood. Lab Chip. 2008 Dec;8(12):2054-61.

13. Sai J, Rogers M, Hockemeyer K, Wikswo JP, Richmond A. Study of chemotaxis and cell-cell interactions in cancer with microfluidic devices. Methods Enzymol. 2016;570:19-45.

14. Haque A, Gheibi P, GaoY, Foster E, Son KJ, You J, et al. Cell biology is different in small volumes: endogenous signals shape phenotype of primary hepatocytes cultured in microfluidic channels. Sci Rep. 2016 Sep;6:33980.

15. Seemann R, Brinkmann M, Pfohl T, Herminghaus S. Droplet based microfluidics. Rep Prog Phys. 2012 Jan;75(1):016601.

16. Sackmann EK, Fulton AL, Beebe DJ.The present and future role of microfluidics in biomedical research. Nature. 2014 Mar;507(7491): 181-9.

17. Olanrewaju A, Beaugrand M, Yafia M, Juncker D. Capillary microfluidics in microchannels: from microfluidic networks to capillaric circuits. Lab Chip. 2018 Aug;18(16):2323-47.

18. Horiuchi T, Hayashi K, Seyama M, Inoue S, Tamechika E. Cooperative suction by vertical capillary array pump for controlling flow profiles of microfluidic sensor chips. Sensors (Basel). 2012 Oct;12(10): 14053-67.

19. Gencturk E, Mutlu S, Ulgen KO. Advances in microfluidic devices made from thermoplastics used in cell biology and analyses. Biomicrofluidics. 2017 Oct;11(5):051502.

20. Shadpour H, Musyimi H, Chen J, Soper SA. Physiochemical properties of various polymer substrates and their effects on microchip electrophoresis performance. J Chromatogr A. 2006 Apr;1111(2): 238-51.

21. Young EW, Berthier E, Beebe DJ.Assessment of enhanced autofluorescence and impact on cell microscopy for microfabricated thermoplastic devices. Anal Chem. 2013 Jan;85(1):44-9.

22. Nie J, Gao Q, Wang Y, Zeng J, Zhao H, Sun Y, et al.Vessel-on-a-chip with Hydrogel-based Microfluidics. Small. 2018 Nov;14(45):e1802368.

23. Zhang X, Li L, Luo C. Gel integration for microfluidic applications. Lab Chip. 2016 May;16(10):1757-76.

24. Iliescu C, Taylor H, Avram M, Miao J, Franssila S. A practical guide for the fabrication of microfluidic devices using glass and silicon. Biomicrofluidics. 2012 Mar;6(1):16505-16.

25. Shin DS, Matharu Z, You J, Siltanen C, Vu T, Raghunathan VK, et al. Sensing conductive hydrogels for rapid detection of cytokines in blood. Adv Healthc Mater. 2016 Mar;5(6):659-64.

26. Yadavali S, Jeong HH, Lee D, Issadore D. Silicon and glass very large scale microfluidic droplet integration for terascale generation of polymer microparticles. Nat Commun. 2018 Mar;9(1):1222.

27. Markov DA, Lillie EM, Garbett SP, McCawley LJ.Variation in diffusion of gases through PDMS due to plasma surface treatment and storage conditions. Biomed Microdevices. 2014 Feb;16(1):91-6.

28. Han P, Bartels DM. Temperature dependence of oxygen diffusion in H2O and D2O. J Phys Chem. 1996 Mar;100(13):5597-602.

29. Unger MA, Chou HP, Thorsen T, Scherer A, Quake SR. Monolithic microfabricated valves and pumps by multilayer soft lithography. Science. 2000 Apr;288(5463):113-6.

30. Bhattacharya S, Datta A, Berg JM, Gangopadhyay S. Studies on surface wettability of poly(dimethyl) siloxane (PDMS) and glass under oxygen-plasma treatment and correlation with bond strength. J Microelectromech Syst. 2005 Jun;14(3):590-7.

31. Gonzalez-Suarez AM, Pena-Del Castillo JG, Hernandez-Cruz A, Garcia-Cordero JL. Dynamic generation of concentration- and temporal-dependent chemical signals in an integrated microfluidic device for single-cell analysis. Anal Chem. 2018 Jul;90(14):8331-6.

32. Park SE, Georgescu A, Huh D. Organoids-on-a-chip. Science. 2019 Jun;364(6444):960-5.

33. Taylor BJ, Howell A, Martin KA, Manage DP, Gordy W, Campbell SD, et al.A lab-on-chip for malaria diagnosis and surveillance. Malar J. 2014 May;13:179.

34. Lau D, Walsh JC, Peng W, Shah VB, Turville S, Jacques DA, et al. Fluorescence biosensor for real-time interaction dynamics of host proteins with HIV-1 capsid tubes. ACS Appl Mater Interfaces. 2019 Sep; 11(38):34586-94.

35. Yeh YT, Gulino K, Zhang Y, Sabestien A, Chou TW, Zhou B, et al. A rapid and label-free platform for virus capture and identification from clinical samples. Proc Natl Acad Sci U S A. 2020 Jan;117(2):895-901.

36. Maerkl SJ. Integration column: microfluidic high-throughput screening. Integr Biol (Camb). 2009 Jan;1(1):19-29. 
37. Volpetti F, Garcia-Cordero J, Maerkl SJ. A microfluidic platform for high-throughput multiplexed protein quantitation. PLoS One. 2015 Feb;10(2):e0117744.

38. Wu J, Dong M, Rigatto C, Liu Y, Lin F. Lab-on-chip technology for chronic disease diagnosis. NPJ Digit Med. 2018 Apr;1:7.

39. KimuraY, Ikeuchi M, Inoue Y, Ikuta K. 3D microdevices that perform sample purification and multiplex qRT-PCR for early cancer detection with confirmation of specific RNAs. Sci Rep. 2018 Nov;8(1): 17480.

40. Kalidoss R, Umapathy S. An overview on the exponential growth of non- invasive diagnosis of diabetes mellitus from exhaled breath by nanostructured metal oxide chemi-resistive gas sensors and $\mu$-preconcentrator. Biomed Microdevices. 2019 Dec;22(1):2.

41. Choi J, Cho SJ, Kim YT, Shin H. Development of a film-based immunochromatographic microfluidic device for malaria diagnosis. Biomed Microdevices. 2019 Aug;21(4):86.

42. Qi R, Zhu G, Wang Y, Wu S, Li S, Zhang D, et al. Microfluidic device for the analysis of MDR cancerous cell-derived exosomes' response to nanotherapy. Biomed Microdevices. 2019 Mar;21(2):35.

43. Wechsler ME, Stephenson RE, Murphy AC, Oldenkamp HF, Singh A, Peppas NA. Engineered microscale hydrogels for drug delivery, cell therapy, and sequencing. Biomed Microdevices. 2019 Mar;21(2):31.

44. Fattahi P, Haque A, Son KJ, Guild J, Revzin A. Microfluidic devices, accumulation of endogenous signals and stem cell fate selection. Differentiation. 2020 Mar-Apr;112:39-46.

45. Mak SY, Li Z, Frere A, Chan TC, Shum HC. Musical interfaces: visualization and reconstruction of music with a microfluidic two-phase flow. Sci Rep. 2014 Oct;4:6675.

46. Kim YH, Ko KP, Kang IG, Jung JH, Oh DK, Jang TY, et al. Low concentration $\mathrm{PM}(10)$ had no effect on nasal symptoms and flow in allergic rhinitis patients. Clin Exp Otorhinolaryngol. 2017 Jun;10(2): 164-7.

47. Khan DA. Allergic rhinitis and asthma: epidemiology and common pathophysiology. Allergy Asthma Proc. 2014 Sep-Oct;35(5):357-61.

48. Sinurat J, Rengganis I, Rumende CM, Harimurti K. Accuracy of serum-specific IgE test with microfluidic array enzyme-linked immunosorbent assay for diagnosing inhalant allergen sensitization in asthma and/or rhinitis allergy patients in Jakarta, Indonesia. Asia Pac Allergy. 2018 Jan;8(1):e10.

49. Huang Z, Luo W, Zou X, Liu X, Cai C, Wu Z, et al. Application of biochip microfluidic technology to detect serum allergen-specific immunoglobulin E (sIgE). JVis Exp. 2019 Apr;(146):e59100.

50. Huang WY, Chou ST, Chen CH, Chou SY, Wu JH, Chen YC, et al. An automatic integrated microfluidic system for allergy microarray chips. Analyst. 2018 May;143(10):2285-92.

51. Zheng L, Fu Y, Jiang X, Man S, Ran W, Feng M, et al. Microfluidic system for high-throughput immunoglobulin-E analysis from clinical serum samples. Talanta. 2015 Oct;143:83-9.

52. Bernstein DI, Schwartz G, Bernstein JA.Allergic rhinitis: mechanisms and treatment. Immunol Allergy Clin North Am. 2016 May;36(2): 261-78.

53. Aljadi Z, Kalm F, Nilsson C, Winqvist O, Russom A, Lundahl J, et al. A novel tool for clinical diagnosis of allergy operating a microfluidic immunoaffinity basophil activation test technique. Clin Immunol. 2019 Dec;209:108268.

54. Hemmings O, Kwok M, McKendry R, Santos AF. Basophil activation test: old and new applications in allergy. Curr Allergy Asthma Rep. 2018 Nov;18(12):77.

55. Czarnobilska EM, Bulanda M, Spiewak R. The usefulness of the basophil activation test in monitoring specific immunotherapy with house dust mite allergens. Postepy Dermatol Alergol. 2018 Feb;35(1): 93-8.

56. Nopp A, Johansson SG, Ankerst J, Bylin G, Cardell LO, Gronneberg $\mathrm{R}$, et al. Basophil allergen threshold sensitivity: a useful approach to
anti-IgE treatment efficacy evaluation. Allergy. 2006 Mar;61(3):298302.

57. Kwok HC, Lau PM,Wu SY, Ho HP, Gao M, Kwan YW, et al. Allergy testing and drug screening on an ITO-coated lab-on-a-disc. Micromachines (Basel). 2016 Feb;7(3):38.

58. Chen QL, Cheung KL, Kong SK, Zhou JQ, Kwan YW, Wong CK, et al. An integrated lab-on-a-disc for automated cell-based allergen screening bioassays. Talanta. 2012 Aug;97:48-54.

59. Aljadi Z, Kalm F, Ramachandraiah H, Nopp A, Lundahl J, Russom A. Microfluidic immunoaffinity basophil activation test for point-ofcare allergy diagnosis. J Appl Lab Med. 2019 Sep;4(2):152-63.

60. Knol EF, Mul FP, Jansen H, Calafat J, Roos D. Monitoring human basophil activation via CD63 monoclonal antibody 435. J Allergy Clin Immunol. 1991 Sep;88(3 Pt 1):328-38.

61. Yan Y, Gordon WM, Wang DY. Nasal epithelial repair and remodeling in physical injury, infection, and inflammatory diseases. Curr Opin Otolaryngol Head Neck Surg. 2013 Jun;21(3):263-70.

62. Na K, Lee M, Shin HW, Chung S. In vitro nasal mucosa gland-like structure formation on a chip. Lab Chip. 2017 May;17(9):1578-84.

63. Yu F, Zhao X, Li C, LiY, Yan Y, Shi L, et al. Airway stem cells: review of potential impact on understanding of upper airway diseases. Laryngoscope. 2012 Jul;122(7):1463-9.

64. Wang W, Yan Y, Li CW, Xia HM, Chao SS, Wang de Y, et al. Live human nasal epithelial cells (hNECs) on chip for in vitro testing of gaseous formaldehyde toxicity via airway delivery. Lab Chip. 2014 Feb; 14(4):677-80.

65. Ayoob AM, Borenstein JT. The role of intracochlear drug delivery devices in the management of inner ear disease. Expert Opin Drug Deliv. 2015 Mar;12(3):465-79.

66. Shi X. Pathophysiology of the cochlear intrastrial fluid-blood barrier (review). Hear Res. 2016 Aug;338:52-63.

67. Tandon V, Kang WS, Spencer AJ, Kim ES, Pararas EE, McKenna MJ, et al. Microfabricated infuse-withdraw micropump component for an integrated inner-ear drug-delivery platform. Biomed Microdevices. 2015 Apr;17(2):37.

68. McCall AA, Swan EE, Borenstein JT, Sewell WF, Kujawa SG, McKenna MJ. Drug delivery for treatment of inner ear disease: current state of knowledge. Ear Hear. 2010 Apr;31(2):156-65.

69. Brown JN, Miller JM, Altschuler RA, Nuttall AL. Osmotic pump implant for chronic infusion of drugs into the inner ear. Hear Res. 1993 Nov;70(2):167-72.

70. Chen Z, Kujawa SG, McKenna MJ, Fiering JO, Mescher MJ, Borenstein JT, et al. Inner ear drug delivery via a reciprocating perfusion system in the guinea pig. J Control Release. 2005 Dec;110(1):1-19.

71. Tandon V, Kang WS, Robbins TA, Spencer AJ, Kim ES, McKenna MJ, et al. Microfabricated reciprocating micropump for intracochlear drug delivery with integrated drug/fluid storage and electronically controlled dosing. Lab Chip. 2016 Mar;16(5):829-46.

72. SewellWF, Borenstein JT, Chen Z, Fiering J, Handzel O, Holmboe M, et al. Development of a microfluidics-based intracochlear drug delivery device. Audiol Neurootol. 2009;14(6):411-22.

73. Jahn K. The Aging vestibular system: dizziness and imbalance in the elderly. Adv Otorhinolaryngol. 2019;82:143-9.

74. Andreou CM, Pahitas Y, Georgiou J. Bio-inspired micro-fluidic angular-rate sensor for vestibular prostheses. Sensors (Basel). $2014 \mathrm{Jul}$; 14(7):13173-85.

75. Nonaka T,Wong DT. Liquid biopsy in head and neck cancer: promises and challenges. J Dent Res. 2018 Jun;97(6):701-8.

76. Denaro N, Merlano MC. Immunotherapy in head and neck squamous cell cancer. Clin Exp Otorhinolaryngol. 2018 Dec;11(4):217-23.

77. Payne K, Brooks J, Spruce R, Batis N,Taylor G, Nankivell P, et al. Circulating tumour cell biomarkers in head and neck cancer: current progress and future prospects. Cancers (Basel). 2019 Aug;11(8):1115.

78. Wang Z, Li F, Rufo J, Chen C, Yang S, Li L, et al. Acoustofluidic sali- 
vary exosome isolation: a liquid biopsy compatible approach for human papillomavirus-associated oropharyngeal cancer detection. J Mol Diagn. 2020 Jan;22(1):50-9.

79. Zhou Q, Rahimian A, Son K, Shin DS, Patel T, Revzin A. Development of an aptasensor for electrochemical detection of exosomes. Methods. 2016 Mar;97:88-93.

80. Contreras-Naranjo JC, Wu HJ, Ugaz VM. Microfluidics for exosome isolation and analysis: enabling liquid biopsy for personalized medicine. Lab Chip. 2017 Oct;17(21):3558-77.

81. Poulet G, Massias J, Taly V. Liquid biopsy: general concepts. Acta Cytol. 2019;63(6):449-55.

82. Paoletti C, Hayes DF. Circulating tumor cells. Adv Exp Med Biol. 2016;882:235-58.

83. Garcia SA, Weitz J, Scholch S. Circulating tumor cells. Methods Mol Biol. 2018;1692:213-9.

84. Cho H, Kim J, Song H, Sohn KY, Jeon M, Han KH. Microfluidic technologies for circulating tumor cell isolation. Analyst. 2018 Jun; 143(13):2936-70.

85. Lin M, Chen JF, LuYT, Zhang Y, Song J, Hou S, et al. Nanostructure embedded microchips for detection, isolation, and characterization of circulating tumor cells. Acc Chem Res. 2014 Oct;47(10):2941-50.

86. Gheibi P, Zeng S, Son KJ, Vu T, Ma AH, Dall'Era MA, et al. Microchamber cultures of bladder cancer: a platform for characterizing drug responsiveness and resistance in PDX and primary cancer cells. Sci Rep. 2017 Sep;7(1):12277.

87. Sontheimer-Phelps A, Hassell BA, Ingber DE. Modelling cancer in microfluidic human organs-on-chips. Nat Rev Cancer. 2019 Feb; 19(2):65-81.

88. Zhang H, ZhuY, Shen Y. Microfluidics for cancer nanomedicine: from fabrication to evaluation. Small. 2018 Jul;14(28):e1800360.

89. Cheah R, Srivastava R, Stafford ND, Beavis AW, Green V, Greenman $\mathrm{J}$. Measuring the response of human head and neck squamous cell carcinoma to irradiation in a microfluidic model allowing customized therapy. Int J Oncol. 2017 Oct;51(4):1227-38.

90. Al-Samadi A, Poor B, Tuomainen K, Liu V, Hyytiainen A, Suleymanova I, et al. In vitro humanized 3D microfluidic chip for testing personalized immunotherapeutics for head and neck cancer patients. Exp Cell Res. 2019 Oct;383(2):111508. 